\title{
Differential Clinical Diagnosis and Prevalence Rate of Allergic Rhinitis, Asthma, and Chronic Obstructive Pulmonary Disease among COVID-19 Patients
}

\author{
Marian Boshra ${ }^{1}$, Mohammad F Mohammad ${ }^{2}$, and Rania Sarhan ${ }^{1}$ \\ ${ }^{1}$ Beni Suef University Faculty of Pharmacy \\ ${ }^{2}$ Beni Suef University Faculty of Medicine
}

February 16, 2021

\begin{abstract}
Background: There is a great need to make a rapid differential clinical diagnosis of COVID-19 among respiratory disease patients and determining the prevalence rate of these diseases among the COVID-19 population. Method: Approximately 522 patients with allergic rhinitis, asthma, COPD, and COVID-19 were analyzed for demographic and clinical features. Radiological features were analyzed only for COVID-19 patients. Results: COPD and asthma were more common among COVID-19 patients than allergic rhinitis. All chest CT scans of COVID-19 patients showed bilateral ground-glass opacity. Fever, dry cough, diarrhea, loss of sense of smell and taste, shortness of breath, and blue lips were significantly higher in all COVID-19 patients compared to COPD, asthma, and allergic rhinitis patients. Conclusion: The presence of clinical symptoms such as fever, dry cough, diarrhea, loss of sense of smell and taste, shortness of breath, and blue lips in COVID-19 patients, can be used for differential diagnosis between COVID-19 patients and other respiratory diseases. Then, the diagnosis can be confirmed by chest CT scan for COVID-19 patients without the need for a nasopharyngeal swab or PCR test, especially in epidemic countries. Allergic rhinitis patients are the least exposed to COVID-19 infection among other respiratory disease patients.
\end{abstract}

Differential Clinical Diagnosis and Prevalence Rate of Allergic Rhinitis, Asthma, and Chronic Obstructive Pulmonary Disease among COVID-19 Patients

${ }^{1}$ Rania M Sarhan, PhD, ${ }^{2}$ Mohammad F Mohammad, PhD, ${ }^{1}$ Marian S. Boshra, PhD

${ }^{1}$ Clinical Pharmacy Department, Faculty of Pharmacy, Beni-Suef University, Beni-Suef, Egypt.

${ }^{2}$ Department of Chest Diseases, Faculty of Medicine, Beni-Suef University, Beni-Suef, Egypt.

\section{Dear editor,}

There is a great interest to make a rapid differential clinical diagnosis of COVID-19 among respiratory disease patients and determining the prevalence rate of these diseases among the COVID-19 population. Symptom screening became a prevalent tool in the attempt to control local dissemination of COVID-19, starting from affected cities to quarantines [1]. This can be attributed to that the coronavirus family may cause varied symptoms such as fever, pneumonia, lung infection, and breathing difficulty [2]. All this has pushed several studies to review the characteristics and symptoms of adults infected with COVID-19 [3]. In our retrospective study, approximately 522 patients with allergic rhinitis, asthma, COPD, and COVID19 were collected from Beni-Suef University hospital and Hospital of Chest Diseases in Beni-Suef, Egypt, and analyzed for demographic and clinical features. Radiological features were analyzed for all COVID-19 patients. About $312(59.8 \%)$ females and 210 (40.2\%) males were included in the study. They were of 
different age groups, 146 (28\%) patients were between the ages of 18 and 30 years, 253 (48.5\%) patients were between the ages of 30 and 45 years, and $123(23.5 \%)$ patients were between the ages of 45 and 60 years.

The patients were grouped into 5 groups: (1) allergic rhinitis patients, (2) asthmatic patients, (3) asthmatic patients with allergic rhinitis, (4) COPD patients, (5) COVID-19 patients. COVID-19 patients were subdivided into 3 groups: (5a) COVID-19 patients only, (5b) COVID-19 patients with COPD, (5c) COVID19 patients with asthma (table 1). Approximately, the prevalence of allergic rhinitis, asthma, and COPD among the 228 participated COVID-19 patients was $0 \%, 30.7 \%$, and $35.1 \%$ respectively (figure 1 ). There was a significant difference in the prevalence of the 3 respiratory diseases among COVID-19 patients. The numbers of COVID-19 patients, having asthma or COPD, were significantly higher compared to the number of COVID-19 patients with allergic rhinitis at $\mathrm{p}<0.05$. There was no significant difference between the number of COPD $(n=80)$ or asthmatic patients $(n=70)$ among COVID-19 patients. All chest CT scans of COVID-19 patients (228 patients, 100\%) showed bilateral ground-glass opacity with abnormal findings on chest CT. Comparing categorical variables between patients groups using the chi-square test showed that symptoms of all COVID-19 patients were significantly different compared to allergic rhinitis, COPD, and asthmatic patients who didn't have COVID-19 infection at $\mathrm{p}<0.05$. Fever, dry cough, diarrhea, loss of smell and taste senses, shortness of breath, and blue lips were significantly higher in all COVID-19 patients at $\mathrm{p}<0.05$ compared to other groups.

We concluded that clinical diagnosis is a very important tool in differentiation between SARS-CoV-2 and other respiratory diseases especially allergic rhinitis, asthma, and COPD. Fever, dry cough, diarrhea, loss of sense of smell and taste, shortness of breath, and blue lips, are the most distinguishing symptoms for COVID-19. After that, COVID-19 diagnosis should be confirmed by ground-glass opacity and abnormal findings on chest CT without the need for a nasopharyngeal swab especially in epidemic countries. Also, COPD and asthmatic patients are more susceptible to COVID-19 infection than allergic rhinitis patients.

\section{Figure legends}

Figure 1: Prevalence rate of allergic rhinitis, asthma, and COPD in COVID-19 population. COPD= Chronic obstructive pulmonary disease, COVID-19= coronavirus disease 2019

* indicates a significant difference between the prevalence rate of asthma and allergic rhinitis in the COVID19 population.

\# indicates a significant difference between the prevalence rate of COPD and allergic rhinitis in the COVID19 population.

\section{References}

1. Gostic, K., et al., Estimated effectiveness of symptom and risk screening to prevent the spread of COVID19. Elife, 2020. 9 : p. e55570.

2. Adhikari, S.P., et al.,Epidemiology, causes, clinical manifestation and diagnosis, prevention and control of coronavirus disease (COVID-19) during the early outbreak period: a scoping review. Infectious diseases of poverty, 2020. 9 (1): p. 1-12.

3. Sun, K., J. Chen, and C. Viboud,Early epidemiological analysis of the coronavirus disease 2019 outbreak based on crowdsourced data: a population-level observational study. The Lancet Digital Health, 2020.

\section{Hosted file}

Table 1.pdf available at https://authorea.com/users/396160/articles/509299-differentialclinical-diagnosis-and-prevalence-rate-of-allergic-rhinitis-asthma-and-chronicobstructive-pulmonary-disease-among-covid-19-patients

\section{Hosted file}


Figure 1.pdf available at https://authorea.com/users/396160/articles/509299-differentialclinical-diagnosis-and-prevalence-rate-of-allergic-rhinitis-asthma-and-chronicobstructive-pulmonary-disease-among-covid-19-patients 